\title{
Damping of simple modes of high-energy nuclear excitations: dispersive optical models and their implementations
}

\author{
M. H. Urin ${ }^{1, a}$ \\ ${ }^{1}$ National Research Nuclear University "MEPhl” (Moscow Engineering Physics Institute), Moscow, Russia
}

\begin{abstract}
The particle-hole dispersive optical model, proposed recently to describe the main properties of high-energy particle-hole-type excitations in medium-heavy mass spherical nuclei, is briefly presented. Unique features of the model, new developments and some implementations are discussed. In applying to the description of deep-hole states, an unitary version of the single-quasiparticle optical model is also briefly presented.
\end{abstract}

Dedicated to the memory of Spartak T. Belyaev

\section{Introduction}

The resonance-like structures, corresponding to simple modes of nuclear excitations (singlequasiparticle and particle-hole-type states), are observed in nuclear reactions at high excitation energies (up to a few tens $\mathrm{MeV}$ ). Therefore, for these energies the mean-field concept does work. In other words, nuclei are "grey" (not "black") for these degrees of freedom. To describe coupling of mentioned high-energy states to many-quasiparticle (chaotic) configurations and also to the singleparticle (s. p.) continuum, phenomenological approaches related to the corresponding optical models seems reasonable to implement in practice. Microscopically-based transition to these models can be performed with the use of the many-body Green-function method introduced in nuclear physics by Migdal [1]. A general consideration of the above-mentioned simple states embedded into continuum and a "sea" of many-quasiparticle configurations has been given in Ref. [2]. It seems, that in applying to medium-heavy mass nuclei, this consideration did not lead to formulation of workable models.

In this talk we present, in the main, the recently developed [3] particle-hole dispersive optical model (PHDOM), its unique features, new developments and some implementations. In applying to the description of deep-hole states, an unitary version of single-quasiparticle dispersive optical model (SQDOM) [4] is also briefly presented.

\section{PHDOM}

\subsection{General description}

The particle-hole dispersive optical model was developed to describe commonly the main relaxation modes of a great variety of high-energy (p-h)-type nuclear excitations (including giant resonances)

a e-mail: urin@theor.mephi.ru 
in "hard" medium-heavy mass spherical nuclei. These relaxation modes include: (i) the p-h strength distribution caused by the shell-structure of nuclei (Landau damping); (ii) coupling to the s. p. continuum (nuclei are open Fermi-systems); (iii) coupling to many-quasiparticle configurations (the spreading effect). Being formulated with the use of Fermi-system Green functions, the model is, actually, an extension of the standard [5] and non-standard [6] continuum-RPA (cRPA) versions to the description of the spreading effect. Within the PHDOM (which is a semi-microscopic model), Landau damping and coupling to the s. p. continuum are described microscopically (in terms of a mean field and the $\mathrm{p}$-h interaction responsible for long-range correlations), while the spreading effect is treated phenomenologically and in average over the energy (in terms of the specific $\mathrm{p}-\mathrm{h}$ interaction, or $\mathrm{p}-\mathrm{h}$ self-energy term). The imaginary part of this self-energy term determines the corresponding real part via the dispersive relationship, which follows from the spectral expansion of the $2 \mathrm{p}-2 \mathrm{~h}$ Green function ( $2 \mathrm{p}-2 \mathrm{~h}$ configurations are doorway-states for the spreading effect).

The unique features of the PHDOM are concerned with its ability to describe (i) the energyaveraged double transition density and, therefore, various strength functions at arbitrary (but highenough) excitation energies, including giant resonances; (ii) direct one-nucleon decay of (p-h)-type excitations, including the so-called "direct+semi-direct" (DSD) reactions induced by a s. p. external field; (iii) the spreading (dispersive) shift of the energy of the resonance structures corresponding to (p-h)-type excitations. In first intensive implementations of the PHDOM [7, 8], the following input quantities were used: (i) the Landau-Migdal p-h interaction responsible for long-range correlations (in Ref. [7], separable isovector velocity-dependent forces were also used); (ii) a phenomenological partially self-consistent mean field (described in details in Ref. [9]); (iii) the properly parameterized imaginary part of the strength of the energy-averaged p-h self-energy term responsible for the spreading effect. Thus, the PHDOM in its implementations seems a rather "economical" model.

Concluding the general description, one can call the PHDOM, as the model of interacting and damping high-energy $\mathrm{p}$-h excitations.

\subsection{Basic relationships (schematically)}

The PHDOM is formulated in terms of energy-averaged quantities. The main one is the $\mathrm{p}$-h Green function (effective p-h propagator) $A\left(x, x^{\prime}, \omega\right)$, where $x$ is the set of $\mathrm{s}$. p. coordinates (including the spin and isospin variables), $\omega$ is the excitation energy. This function satisfy the Bethe-Goldstone-type equation:

$$
A(\omega)=A_{0}(\omega)+\left[A_{0}(\omega) F A(\omega)\right] .
$$

Here, $A_{0}$ is the "free" $\mathrm{p}$-h propagator, $F\left(x, x^{\prime}\right)$ is a $\mathrm{p}$-h interaction responsible for long-range correlations, the brackets $[\ldots]$ mean proper integrations. The expression for the key quantity, $A_{0}\left(x, x^{\prime}, \omega\right)$, was derived with the use of the statistical assumption: after energy averaging different $\mathrm{p}$-h excitations (having the same quantum numbers) are "decaying" into chaotic states independently of one another. Let $H_{0}(x)$ be a s. p. Hamiltonian, which determines sets of the s. p. energies $\left\{\varepsilon_{\lambda}\right\}$ and corresponding wave functions $\left\{\phi_{\lambda}\right\}$. The below-given expression for $A_{0}$ is a direct extension of that exploited within the discrete-RPA (" $\lambda$ - representation"):

$$
\begin{gathered}
A_{0}\left(x, x^{\prime}, \omega\right)=\sum_{\lambda, \mu} A_{0, \lambda \mu} \phi_{\mu}^{*}(x) \phi_{\lambda}(x) \phi_{\lambda}^{*}\left(x^{\prime}\right) \phi_{\mu}\left(x^{\prime}\right) ; \\
A_{0, \lambda \mu}=\frac{n_{\lambda}-n_{\mu}}{\varepsilon_{\lambda}-\varepsilon_{\mu}-\omega+\left(n_{\lambda}-n_{\mu}\right)(i W(\omega)-P(\omega)) f_{\lambda} f_{\mu}} .
\end{gathered}
$$

Here, $n_{\lambda, \mu}$ are the occupation numbers, $(-i W(\omega)+P(\omega))$ is the strength of the properly parameterized energy-averaged $\mathrm{p}$-h self-energy term, $f_{\lambda, \mu}$ are the diagonal matrix elements of the Woods-Saxon 
function $f(x)$. Being the phenomenological quantity, the imaginary part of the mentioned strength, $W(\omega)$, determines the real part, $P(\omega)$, via the proper dispersive relationship [3,10].

The comments to derivation of the basic expression (2) are the following [3]. To realize the abovementioned statistical assumption, we start from the integral equation for the non-local (non-averaged) "free" p-h Green function $\mathcal{A}_{0}\left(x, x_{1} ; x^{\prime}, x_{1}^{\prime} ; \omega\right): \mathcal{A}_{0}(\omega)=\mathcal{A}_{0}^{(0)}(\omega)+\left[\mathcal{A}_{0}^{(0)} \Pi(\omega) \mathcal{A}_{0}(\omega)\right]$. Here, $\mathcal{A}_{0}^{(0)}$ is the free $\mathrm{p}$-h propagator, $\Pi\left(x, x_{1} ; x^{\prime}, x_{1}^{\prime} ; \omega\right)$ is the self-energy term. The spectral expansion for $\mathcal{A}_{0}^{(0)}$ contains the $\mathrm{p}$-h transition-density matrices $\rho_{\lambda \mu}^{(0)}\left(x, x_{1}\right)=\left(1-n_{\lambda}\right) n_{\mu} \phi_{\lambda}^{*}(x) \phi_{\mu}\left(x_{1}\right)$. Differently from the transition densities $\rho_{\lambda \mu}^{(0)}(x)=\rho_{\lambda \mu}^{(0)}\left(x=x_{1}\right)$, the transition-density matrices are orthogonal: $\left[\rho_{\lambda \mu}^{(0)} \rho_{\lambda^{\prime} \mu^{\prime}}^{(0)}\right]=\delta_{\lambda \lambda^{\prime}} \delta_{\mu \mu^{\prime}}$. This point allows one to realize the statistical assumption, using the proper parametrization of the energy-averaged self-energy term: $\Pi\left(x, x_{1} ; x^{\prime}, x_{1}^{\prime} ; \omega\right) \rightarrow(-i W(\omega)+P(\omega)) f(x) f\left(x_{1}\right) \delta\left(x-x^{\prime}\right) \delta\left(x_{1}-x_{1}^{\prime}\right)$. Then the equation for the energy-averaged "free" $\mathrm{p}$-h non-local Green function, $A_{0}\left(x, x_{1} ; x^{\prime}, x_{1}^{\prime} ; \omega\right)$, can be approximately solved. In the local limit $\left(x=x^{\prime}, x_{1}=x_{1}^{\prime}\right)$, the obtained quantity is given by the expression (2).

With the use of the approximate spectral expansion for the Green functions $g\left(x, x^{\prime}, \varepsilon=\varepsilon_{v} \pm\right.$ $\omega)$ of the s. p. Schrodinger equation, which contains the addition to the mean field $[-i W(\omega)+$ $P(\omega)] f_{v} f(x)$, one can transform the expression (2) for $A_{0}\left(x, x^{\prime}, \omega\right)$ to the form, allowing to take the s. p. continuum into account. (Within the cRPA, this transformation is exact). The continuum-state s. p. wave functions $\phi_{\varepsilon}^{( \pm)}$, satisfying to the mentioned Schrodinger equation, are also used in the continuum version of the PHDOM. One can say, that within this version, there appears the effective optical-model potential. The imaginary part of this potential (adjusted to describe the observable total width of various giant resonances) was found noticeably less, than the imaginary part used within the ordinary optical model to describe nucleon-nucleus scattering at the corresponding energies. Such a conclusion is in agreement with theoretical considerations of this problem (see, e. g., Ref. [11]).

Within the model, a version of the dispersive relationship (adopted to satisfy the condition $P(\omega \rightarrow$ 0) $\rightarrow 0$ )

$$
P(\omega)=\frac{2}{\pi} P . V . \int d \omega^{\prime} W\left(\omega^{\prime}\right)\left(\frac{\omega^{\prime}}{\omega^{2}-\omega^{\prime 2}}+\frac{1}{\omega^{\prime}}\right)
$$

can be used together with the simplest parameterization of $W: 2 W(\omega)=\alpha \omega^{2} /\left(1+(\omega / B)^{2}\right)[10]$. Here, the strength parameter $\alpha$ and "saturation" parameter $B$ are adjustable quantities (a saturationlike energy dependence of $W(\omega)$ follows from the above-mentioned analysis of the total width of various giant resonances). As a result, one can get from Eq. (3): $P(\omega)=(2 / \pi) W(\omega) \ln (\omega / B)$. In implementations of the PHDOM [7, 8], for $W(\omega)$ we use the more sophisticated parameterization, which contains also an adjustable "gap" parameter $\Delta$. (It is supposed, that the spreading effect appears at a finite excitation energy $\omega_{\min }=\Delta$ ). The dispersive relationship is properly adopted $[3,10]$.

Expressions for main PHDOM quantities are derived, starting from the p-h Green function of Eqs. (1), (2). In particular, the energy-averaged double transition density $\rho\left(x, x^{\prime}, \omega\right)$ and strength function $S_{V_{0}}(\omega)$, related to a s. p. external field (probing operator) $V_{0}(x)$, are:

$$
\begin{aligned}
\rho\left(x, x^{\prime}, \omega\right) & =-\frac{1}{\pi} \operatorname{Im} A\left(x, x^{\prime}, \omega\right), \\
S_{V_{0}}(\omega) & =-\frac{1}{\pi} \operatorname{Im}\left[V_{0}^{+} A V_{0}\right] .
\end{aligned}
$$

These quantities can be evaluated at arbitrary (but high-enough) excitation energies, including distant "tails" of giant resonances. It is quite reasonable, that, within the model, the energy-averaged double transition density cannot be factorized. In other words, one-body transition density cannot be defined with taking the spreading effect into account. Since the computer codes developed for describing the 
cross sections of inelastic hadron-nucleus scattering accompanied by excitation of a given giant resonance exploit only one-body transition density, we suggest to use for these codes the corresponding energy-dependent "projected" transition density $\rho_{g}(x, \omega)$ defined as follows [8]:

$$
\rho_{g}(x, \omega)=\frac{\int \rho\left(x, x^{\prime}, \omega\right) V_{g, 0}\left(x^{\prime}\right) d x^{\prime}}{\left(S_{V_{g, 0}}(\omega)\right)^{1 / 2}} .
$$

Here, $V_{g, 0}(x)$ is the specific for excitation of a given giant resonance probing operator. The giantresonance strength function is determined, as it follows from Eqs. (4), (5), by the "projected" transition density: $S_{V_{g, 0}}(\omega)=\left|\left(\rho_{g}(\omega) V_{g, 0}\right)\right|^{2}$.

Being proposed in Ref. [1], the effective-field method, which allows one to simplify calculations of some observables, can be also used within the PHDOM. Related to a s. p. probing operator $V_{0}(x)$ the energy-averaged effective field $V(x, \omega)$ is defined by the relationship: $\left[A(\omega) V_{0}\right]=\left[A_{0}(\omega) V(\omega)\right]$. In accordance with Eqs. (1) and (5), the effective field satisfies the equation

$$
V(\omega)=V_{0}+\left[F A_{0}(\omega) V(\omega)\right],
$$

and determines the strength function $S_{V_{0}}(\omega)=-1 / \pi \operatorname{Im}\left[V_{0}^{+} A_{0}(\omega) V(\omega)\right]$.

The effective-field method allows one to get within the PHDOM the expression for the squared energy-averaged amplitude of one-nucleon direct+semi-direct (DSD) reaction induced by a s. p. external field $V_{0}(x)$. Being an extension of the corresponding relationship obtained within the cRPA [6], this expression might be presented in the form [12]:

$$
\left|M_{V_{0}, c}^{D S D}(\omega)\right|^{2}=n_{\mu}\left|\left[\phi_{\varepsilon}^{(-) *} V(\omega) \phi_{\mu}\right]\left[\phi_{\mu}^{*} V^{*}(\omega) \phi_{\varepsilon}^{(+)}\right]\right| .
$$

Here, $c$ is the set of reaction-channel quantum numbers, that includes the quantum numbers of the s. p. continuum state $\left(\varepsilon=\varepsilon_{\mu}+\omega\right), \mu^{-1}$ state populated in the DSD-reaction, and of (p-h)-type nuclear excitation. The simplified form of the expression (8), in which the optical-model strength function $\phi_{\varepsilon}^{(+)}$is used instead of $\phi_{\varepsilon}^{(-) *}$ has been proposed in Ref. [3]. Such a substitution is valid only within the cRPA. The expression (8) might be used to evaluate partial branching ratios for direct one-nucleon decay of a given giant resonance:

$$
b_{g, c}(\delta)=\frac{\int_{(\delta)}\left|M_{V_{g, 0}, c}^{D S D}(\omega)\right|^{2} d \omega}{\int_{(\delta)} S_{V_{g, 0}}(\omega) d \omega} .
$$

Here, the energy interval $\delta$ includes the giant resonance. Within the cRPA, the total direct-nucleondecay branching ratio equals to unity independently of the choice of the energy interval and external field. There is an alternative way for evaluation of the partial branching ratios, which consists in calculating channel matrix elements of the energy-averaged scattering amplitude $\Gamma\left(x, x^{\prime}, \omega\right)$. The latter is defined, as follows: $[A(\omega) F]=\left[A_{0}(\omega) \Gamma(\omega)\right]$ [3]. Within the cRPA, such a method was used in Ref.[13].

\subsection{Unitary version}

The methods used within the PHDOM for the description of the spreading effect lead to weak violations of model unitarity. The sources of these violations are an energy dependence of the p-h selfenergy term, and the approximate spectral expansion of the optical-model Green function. The signatures of unitarity violations within the PHDOM are the appearance of: (i) nonzero values (markedly 
larger than uncertainties of numerical calculations) of the spurious strength function, corresponding to the unit external field; (ii) small negative values of the strength function of the isoscalar giant monopole resonance (ISGMR) at the energies much larger than the ISGMR energy. The last effect leads to an underestimation of the total ISGMR strength. The above-described problems have been investigated in Ref. [12], where the methods for unitarity restoration have also been proposed.

Within the discrete-PHDOM version, the unitarity violation caused by an energy-dependence of the self-energy term can be approximately restored by the following modification of the expression (2):

$$
A_{0, \lambda \mu}(\omega) \rightarrow A_{0, \lambda \mu}^{m}(\omega)=A_{0, \lambda \mu}(\omega)\left(1+\left(n_{\lambda}-n_{\mu}\right) \frac{d P}{d \omega} f_{\lambda} f_{\mu}\right) .
$$

(Contributions of terms proportional to $d^{2} W / d \omega^{2}$ can be neglected). As it follows from the expressions (2), (10), within the modified discrete-PHDOM version spurious (isoscalar monopole (ISM)) transitions induced by the unit external field are strictly forbidden. The approximate transformation of $A_{0}^{m}\left(x, x^{\prime}, \omega\right)$ to the expression, in which the s. p. continuum is taken into account, leads to appearance of properly modified optical-model Green functions and continuum-state wave functions.

Spurious contributions appear in the description of ISM excitations within the initial and modified continuum-PHDOM versions due to the use of the approximate spectral expansion of the opticalmodel Green functions. These contributions can be eliminated, if the isoscalar external field $V_{0}(r) Y_{00}$ is renormalized to get correspondingly the renormalized and unitary continuum-PHDOM versions:

$$
V_{0}(r) \rightarrow V_{0}(r)-\left\langle V_{0}\right\rangle
$$

Here, averaging is performed over the ground-state radial matter density. To illustrate the briefly described above methods of unitarity restoration (given in details in Ref. [12]), one can consider the relative energy-weighted ISM strength functions $y_{g}(\omega)=\omega S_{V_{g, 0}}(\omega) / E W S R_{g}$ with $E W S R_{g}$ being the energy-weighted sum rule for the external field $V_{g, 0}(r) Y_{00}$. In Fig. 1, the relative strength functions calculated for the ISGMR $\left(g=1, V_{1,0}=r^{2}\right)$ in ${ }^{208} \mathrm{~Pb}$ within the initial and unitary PHDOM versions are shown. Different stages of unitarity restoration are illustrated by the integrated features $x_{g}=$ $\int y_{g}(\omega) d \omega$ calculated for the same giant resonance within the cRPA, initial, modified, renormalized, and unitary continuum-PHDOM versions (Table 1). In conclusion, violations of model unitarity are found to be weak and can be reasonably eliminated.

\subsection{Implementations}

The first intensive implementation of the PHDOM was concerned with the description of simplest photonuclear reactions (photo-absorption, $\operatorname{DSD}(\gamma, n)$ and inverse reactions) accompanied by excitation of the isovector giant dipole and quadrupole resonances (IVGDR and IVGQR, respectively) [7]. In such a case, the external fields are well-known and PHDOM equations can be directly exploited. However, if the Landau-Migdal forces are only used, the IVGDR energy and photo-absorption cross section integrated over the resonance are found to be markedly underestimated within the model. This point forced us to use also velocity-dependent forces (taken in the simplest (separable) form with dimensionless strengths $k_{1}^{\prime}$ and $k_{2}^{\prime}$ ). Two phenomenological parameters $\alpha$ and $k_{1}^{\prime}$ were adjusted to describe the IVGDR energy and total width for a few singly- and doubly-closed-shell medium-heavy mass nuclei. (Since other model parameters are taken from independent data, the reasonable description of the experimental photo-absorption cross section integrated over the IVGDR in ${ }^{208} \mathrm{~Pb}$ is an evidence of consistency of the model). Then the differential partial DSD $(n, \gamma)$-reaction cross sections have been satisfactory described for mentioned nuclei without the use of new adjustable parameters. The appropriate subject for studying the IVGQR in photonuclear reactions is the asymmetry (with 


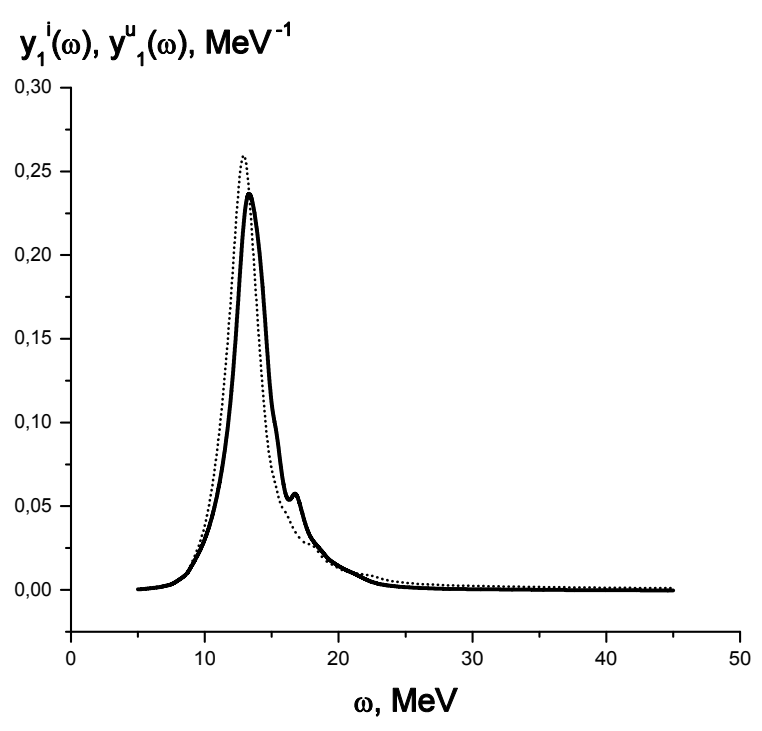

Figure 1. The relative energy-weighted strength functions calculated for the ISGMR in ${ }^{208} \mathrm{~Pb}$ within the initial (thick line) and unitary (dotted line) versions of the PHDOM.

respect to $\left.90^{\circ}\right)$ of the differential $\operatorname{DSD}(\gamma, n)$ - and $(n, \gamma)$-reaction cross sections. The asymmetry value is proportional to the product of $E 1$ - and $E 2$-reaction amplitudes and, therefore, exhibits a non-regular energy behavior in the IVGQR region. (In such a case, the E1-amplitude is taken at the distant "tail" of the IVGDR). To describe better the experimental data on asymmetry values for target nuclei around $A=208$, we adjust properly the "velocity" parameter $k_{2}^{\prime}$. It allowed us also to describe the IVGQR strength function for these nuclei without the use of free parameters. Further studies of simplest photonuclear reactions within the PHDOM are supposed to be related to an improvement of the description of DSD-reaction amplitudes and consideration of contribution of the $\operatorname{DSD}(\gamma, n)$-reaction in photo-absorption accompanied by escaping only one neutron. The corresponding experimental data are available.

Another implementation of the PHDOM is concerned with the description of high-energy ISM excitations [8]. The interest in experimental and theoretical studies of these excitations is mainly due to the possibility of determining the nuclear matter incompressibility coefficient. The latter depends on the mean energy of the ISGMR. To deduce the respective strength distribution from experimental cross sections of $\left(\alpha, \alpha^{\prime}\right)$-scattering at small angles, the properly normalized classical collective-model (energy-independent, one-body) transition density of the ISGMR is usually used within the DWBAbased analysis of the cross sections (see, e. g., Ref. [14]). Within a microscopic approach, the input quantity for the analysis of the $\left(\alpha, \alpha^{\prime}\right)$-reaction cross section should be the energy-averaged double transition density. In applying to high-energy ISM excitations in ${ }^{208} \mathrm{~Pb}$, the radial (two-dimensional) double transition density, $\rho\left(r, r^{\prime}, \omega\right)$, was studied in details within the initial PHDOM version [8]. The properly normalized energy-weighted ISGMR strength function $y_{1}^{i}(\omega)$ calculated in accordance with Eqs. (4), (5) is shown in Fig. 1. Properties of the ISMGR2 (overtone of the ISGMR) were also studied in Refs. $[8,12]$. Apart from the study of $\rho\left(r, r^{\prime}, \omega\right)$, the possibility to factorize this quantity in terms 


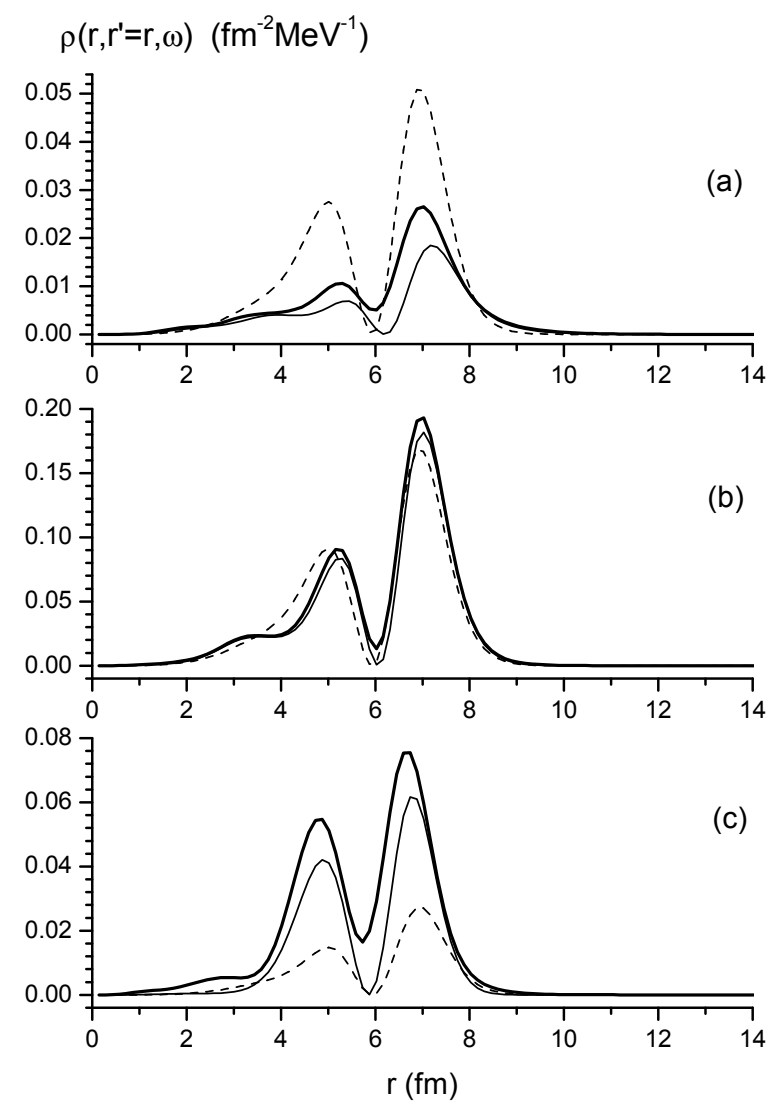

Figure 2. The ISM double transition density $\rho(r, r, \omega)$ calculated at the different excitation energies in a vicinity of the ISGMR: $10.8 \mathrm{MeV}$ (a), 13.8 MeV (b), $16.8 \mathrm{MeV}$ (c) (the solid thick lines) in a comparison with the factorized projected and microscopically corrected classical double transition densities, $\rho_{1}(r, r, \omega)$ (the solid thin and dashed lines, respectively).

of the projected and microscopically corrected classical (radial, one-dimensional) transition densities, $\rho_{1}(r, \omega)$ (see Eq. (6)) and $\rho_{c, 1}^{\Lambda}(r, \omega)$, respectively, was investigated in Ref. [8]. The corrected classical density is defined as follows:

$$
\rho_{c, 1}^{\Lambda}(r, \omega)=\Lambda^{1 / 2}(\omega) \rho_{c, 1}(r), \quad \Lambda(\omega)=\frac{S_{V_{1,0}}(\omega)}{\left[\rho_{c, 1} V_{1,0}\right]^{2}} .
$$

Here, $\rho_{c, 1}(r) \sim\left(3+r \frac{d}{d r}\right) n(r)$ is the classical collective-model transition density normalized to the $E W S R_{1}(n(r)$ is the ground-state matter density). In Fig. 2, the calculated "diagonal" double transition density $\rho\left(r, r^{\prime}=r, \omega\right)$ is compared with the squared radial projected and microscopically corrected classical transition densities at the energies corresponding to the ISGMR maximum and its energy "tails". From this comparison it follows, in particular, that the substitution of the double transition density by the squared projected transition density is better than the substitution by the squared 


\begin{tabular}{|c|c|c|c|c|}
\hline$x_{1}^{c R A}$ & $x_{1}^{i}$ & $x_{1}^{m}$ & $x_{1}^{r n}$ & $x_{1}^{u}$ \\
\hline 0.987 & 0.974 & 0.878 & 1.046 & 1.000 \\
\hline
\end{tabular}

Table 1. Integrated features of ISMGR calculated in various approximations.

microscopically corrected classical transition density. The similar conclusion was obtained in Ref. [8] from consideration of the ISGMR strength function related to the external field $V_{q, 0}(r)=\sin q r / q r$, where $\hbar q$ is momentum transfer at zero-angle $\left(\alpha, \alpha^{\prime}\right)$-scattering accompanied by ISGMR excitation. Being calculated in the Born approximation, the cross section of this scattering is proportional to the above-mentioned strength function. The partial and total branching ratios for direct neutron decay of the ISGMR in ${ }^{208} \mathrm{~Pb}$ were also studied within the PHDOM [12]. The total branching ratio calculated without the use of specific adjustable parameters was found to be about two times larger than the corresponding experimental value. The reasons for this disagreement are not clear now. As a nearest step in investigations of isoscalar excitations within the PHDOM, we plan to incorporate the ISGMR projected transition density in computer codes for calculating the cross sections of inelastic $\alpha$-scattering at small angles. Another step is concerned with investigation of isoscalar multipole giant resonances $(L=1-3)$ studied experimentally.

One further implementation of the PHDOM is a quantitative estimation of the spreading width of the Isobaric Analog Resonances (IARs), $\Gamma_{A}^{\downarrow}$. The small value of this width (several tens keV) is the impressive manifestation of the approximate isospin-symmetry conservation in medium-heavy mass nuclei. For the IARs, the spreading effect is strongly suppressed and realized due to isospin mixing. In mentioned nuclei, the main mixing mechanism consists in coupling the IAR to its overtone via a variable part of the mean Coulomb field $U_{C}(r)$ (The IAR overtone is the charge-exchange giant monopole resonance in the $\beta^{-}$-channel $\left(\operatorname{IVGMR}^{(-)}\right)$). To get a quantitative estimation of $\Gamma_{A}^{\downarrow}$, we turn to the "Coulomb description" of IAR properties [15], which allows one to express the IAR total width $\Gamma_{A}$ in terms of the energy-averaged strength function related to the external field $V_{0}(x) \rightarrow V_{C}^{(-)}=$ $\left(U_{C}(r)-\omega_{A}+(i / 2) \Gamma_{A}\right) \tau^{(-)}$:

$$
\Gamma_{A}=\frac{2 \pi}{S_{A}} S_{V_{C}^{(-)}}\left(\omega=\omega_{A}\right)
$$

Here, $S_{A}$ is the IAR Fermi strength (close to $(N-Z)$ ), $\omega_{A}$ is the IAR excitation energy counted off the parent-nucleus ground-state energy. With reasonable accuracy these quantities can be found from the Fermi strength function (related to the probing operator $V_{0}(x) \rightarrow \tau^{(-)}$) calculated within cRPA. In this approximation the IAR total width is determined by the transcendental Eq. (13). Since the IAR is located at the distant low-energy "tail" of the $\operatorname{IVGMR}^{(-)}$, it is quite appropriate to use the PHDOM for evaluation of the above-mentioned Coulomb strength function and also of the squared Coulomb "DSD-reaction" amplitudes. The latter determine the IAR partial and total proton-escape widths:

$$
\Gamma_{A}^{\uparrow}=\sum_{v} \Gamma_{A, v}^{\uparrow}=\frac{2 \pi}{S_{A}} \sum_{v}\left|M_{V_{C}^{(-)}, v}^{D S D}\left(\omega=\omega_{A}\right)\right|^{2} .
$$

Then, the IAR spreading width can be found as the difference $\Gamma_{A}-\Gamma_{A}^{\uparrow}=\Gamma_{A}^{\downarrow}$. Two "spreading" parameters, $\alpha$ and $B$, which determine the $W(\omega)$ energy dependence (Subsection 2.2) are adjusted to describe the observable energy and total width of the $\operatorname{IVGMR}^{(-)}$in the ${ }^{208} \mathrm{~Pb}$ parent nucleus. Such a description can be obtained within the PHDOM by calculating the respective monopole strength function. Then, the IAR spreading width is quantitatively estimated within the proposed combined approach without the use of adjustible parameters [16]. The energy-averaged Coulomb strength calculated for the ${ }^{208} \mathrm{~Pb}$ 


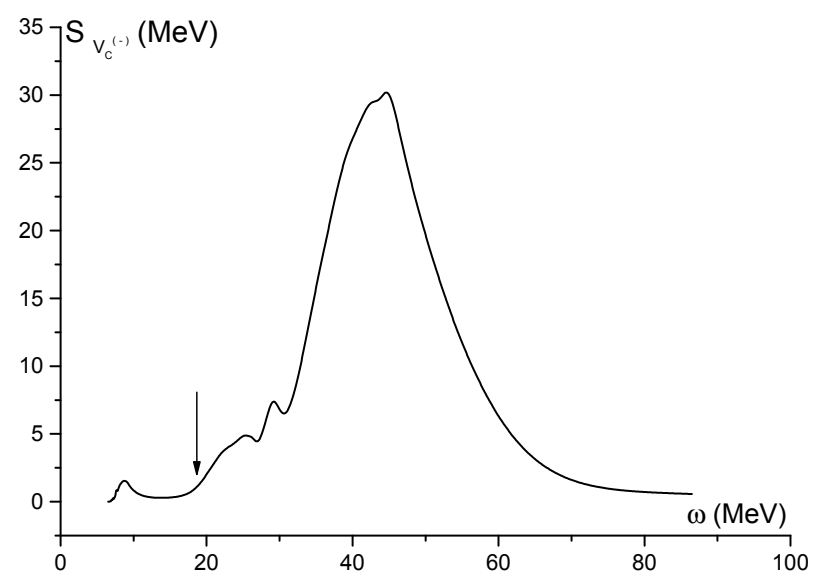

Figure 3. The energy-averaged Coulomb strength function $S_{V_{C}^{(-)}}(\omega)$. The arrow marks the IAR excitation energy $\omega_{A}$.

parent nucleus in a wide excitation energy interval, that includes the IAR and $\operatorname{IVGMR}^{(-)}$, is shown in Fig. 3. As expected, no resonance structure was found at the IAR energy, $\omega_{A}$. The first result obtained for this nucleus allows us to hope that the old theoretical problem - a quantitative estimation of the IAR spreading width - might be solved, if the "Coulomb description" of IAR properties is incorporated into the PHDOM. In such a case, this combined approach is expected to be applied also to the description of: (i) the IAR partial proton-escape widths; (ii) an IAR asymmetry in the excitation functions of proton-induced reactions; (iii) the partial branching ratios for direct proton (neutron) decay of the $\operatorname{IVGMR}^{(-)}\left(\operatorname{IVGMR}^{(+)}\right)$.

\subsection{Summary and conclusive remarks}

The new semi-microscopic model, that takes commonly into account the main relaxation modes of high-energy (p-h)-type nuclear excitations in medium-heavy mass "hard" spherical nuclei is briefly presented. The unique feature of the model is its ability to describe the energy-averaged double transition density (and, therefore, various strength functions) at arbitrary (but high-enough) excitation energy, and also direct-nucleon-decay properties of mentioned excitations. Weak violations of model unitarity were examined and methods of unitarity restoration were proposed. Implementations of the model to the description of simplest photonuclear reactions, isoscalar monopole excitations, the spreading width of Isobaric Analog Resonances are briefly discussed together with new subjects for investigations. Further development of the model might be related to the use of a more realistic mean field and $\mathrm{p}$-h interaction responsible for long-range correlations and (or) taking nucleon pairing into account for open-shell nuclei.

\section{SQDOM}

The single-quasiparticle optical model was first proposed to describe nucleon scattering by mediumheavy mass spherical nuclei. Being used within the model, the Schrödinger equation for the s. p. 
continuum-state wave function contains an energy-dependent complex-valued potential. A few systematics of parameters of this potential are widely used by experimentalists. The model was extended for negative s. p. energies to describe also deep-hole states, and the formally derived dispersive relationship for the spreading (real) part of the mentioned potential was proposed in Ref. [17] to formulate a version of the single-quasiparticle dispersive optical model (SQDOM).

The microscopically-based transition to the SQDOM was proposed in Ref. [9] by energyaveraging the Dyson equation for the single-quasiparticle Fermi-system Green function. Such a way leads to formulation of the SQDOM in terms of the Green function (GF) of the respective Schrödinger equation, while the dispersive relationship follows from the spectral expansion of the 3-quasiparticle Green function. For spherical nuclei, one can consider the radial GFs, $g_{(\lambda)}\left(r, r^{\prime}, \varepsilon\right)$, where $(\lambda)=j_{\lambda}, l_{\lambda},|\varepsilon-\mu|=|E|$ is the quasiparticle excitation energy ( $\mu$ is the chemical potential). The consideration of deep-hole states (i. e. of the discrete-state problem, when $\varepsilon<\mu$ ) allows one to understand the sources and scale of unitarity violation of the model. The main source is the energy-dependent real part of the optical-model potential, $\Delta^{e f f}(r, \varepsilon)=\Delta^{e f f}(\varepsilon) f(r)$ (In further consideration, the total real part contains also the energy-independent mean field used in implementations of the PHDOM). The integral strength of deep-hole states (spectroscopic factor), $S_{\lambda}=(1 / \pi) \operatorname{Im} \int g_{(\lambda)}\left(r=r^{\prime}, \varepsilon \rightarrow \varepsilon_{\lambda}\right) d r d \varepsilon=\int S_{\lambda}(\varepsilon) d \varepsilon$, was evaluated for neutron and proton one-hole states in the parent nuclei ${ }^{90} \mathrm{Zr}$ and ${ }^{208} \mathrm{~Pb}$, neglecting by the imaginary part of the optical-model potential [9]. The strength and energy dependence of $\Delta^{e f f}(\varepsilon)$ were adjusted to reproduce in calculations the observed one-hole energies $\varepsilon_{\lambda}$, using the Schrödinger equation, which contains the energy-dependent part of the full potential. The calculated $S_{\lambda}$ values are found to be $2-3$ times larger than unity that means strong violation of number-of-particle conservation.

This point forced us first to restore model unitarity on the "potential" level (i. e. in ignoring the spreading effect), when $\Delta^{e f f}(\varepsilon)=\Delta^{p}(\varepsilon)$. The mean-field energy-dependent part $\Delta^{p}(\varepsilon) f(r)$ simulates the corresponding results of various Hartree-Fock-type calculations. Above-mentioned restoration can be obtained through the substitution of the "potential" GF $g_{(\lambda)}^{p}\left(r, r^{\prime}, \varepsilon\right)$ by the properly modified GF $[4,18]$ :

$$
g_{(\lambda)}^{p, m}\left(r, r^{\prime}, \varepsilon\right)=\left(q^{p}(r, \varepsilon)\right)^{1 / 2} g_{(\lambda)}^{p}\left(r, r^{\prime}, \varepsilon\right)\left(q^{p}\left(r^{\prime}, \varepsilon\right)\right)^{1 / 2} .
$$

Here, $q^{p}(r, \varepsilon)=\left(1-\frac{d \Delta^{p}(\varepsilon)}{d \varepsilon}\right) f(r)$. Being determined by the GF (15), the corresponding one-hole strengths are found to be close to unity.

Taking the spreading effect into account through energy averaging the Dyson equation, we use the GF of Eq. (15), as the free term [4]. In this case, the integral equation for the optical-model GF schematically looks as follows: $g=g^{p, m}+\left[g^{p, m} \Sigma g\right]$. Here, $\Sigma(r, \varepsilon)=\left(i W(\varepsilon)+\Delta^{d}(\varepsilon)\right) f(r)$ is the strength of the properly parameterized energy-averaged single-quasiparticle self-energy term taken for $\varepsilon<\mu$. The imaginary part of $\Sigma$ (taken at the arbitrary energy) determines the real part via a dispersive relationship. The simplest version of this relationship is the following $[4,9,18]$ :

$$
\Delta^{d}(\varepsilon)=\frac{2 E}{\pi} P . V . \int_{0}^{\infty} \frac{W\left(E^{\prime}\right)}{E^{2}-E^{\prime 2}} d E^{\prime}
$$

where $E=\varepsilon-\mu$. The formal solution of the above-given integral equation for the optical-model GF can be presented in the form:

$$
g_{(\lambda)}\left(r, r^{\prime}, \varepsilon\right)=\left(q ^ { p } ( r , \varepsilon ) ^ { 1 / 2 } g _ { ( \lambda ) } ^ { a u x } ( r , r ^ { \prime } , \varepsilon ) \left(q^{p}\left(r^{\prime}, \varepsilon\right)^{1 / 2}\right.\right.
$$




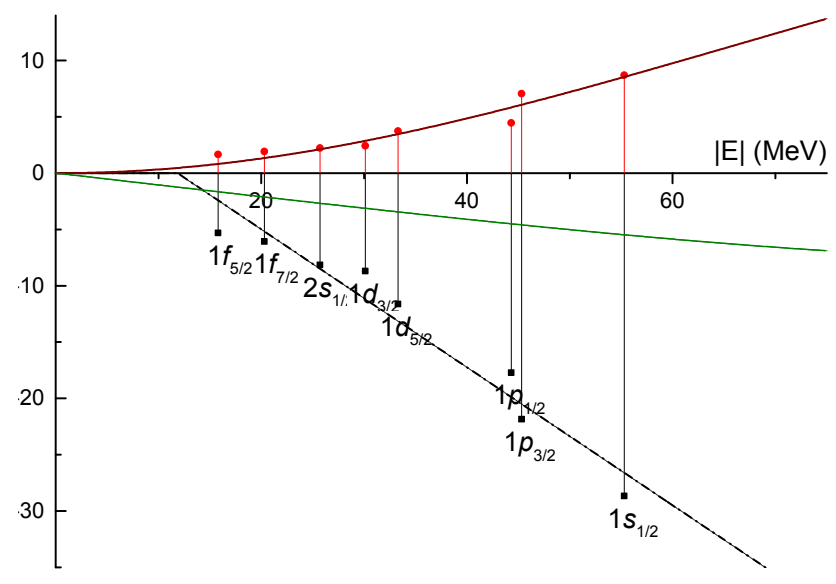

Figure 4. Values found for $W\left(\varepsilon_{\lambda}\right)(\bullet)$ and $\Delta^{e f f}\left(\varepsilon_{\lambda}\right)(\bullet)$ from experimental data. The thick solid red, thin solid green, and dash-dotted black curves represent the results of our calculations for $W(\varepsilon), \Delta^{d}(\varepsilon) q^{p}(\varepsilon)$, and $\Delta^{e f f}(\varepsilon)$, respectively.

Here, $g_{(\lambda)}^{a u x}$ is the GF of the Schrodinger equation, which contains the energy-dependent addition to the mean field $i W^{e f f}+\Delta^{e f f}$ :

$$
\begin{array}{r}
\Delta^{e f f}(r, \varepsilon)=\Delta^{p}(r, \varepsilon)+\Delta^{d}(r, \varepsilon) q^{p}(r, \varepsilon), \\
W^{e f f}(r, \varepsilon)=W(r, \varepsilon) q^{p}(r, \varepsilon) .
\end{array}
$$

Thus, the "spreading" quantities $W(r, \varepsilon)$ and $\Delta^{d}(r, \varepsilon)$ are renormalized due to unitarity restoration on the "potential" level. In particular, the energy-dependent real part of the optical-model potential cannot be presented as the sum of the potential and dispersive terms. Such a sum is exploited in the SQDOM version proposed in Ref. [17].

Since the observable single-hole resonance structures are not overlapped (i. e. the W value is not too large), the equation for GF $g_{(\lambda)}^{\text {aux }}\left(r, r^{\prime}, \varepsilon\right)$ can be solved in the pole approximation. As a result, the one-hole strength function $S_{\lambda}(\varepsilon)$ can be found. In some simplifying (but realistic) approximations, the strength function can be described by a Lorentzian, whose width $\Gamma_{\lambda}$ and integral strength $S_{\lambda}$ are:

$$
\begin{array}{r}
\Gamma_{\lambda}=2 W_{\lambda \lambda}\left(\varepsilon_{\lambda}\right) S_{\lambda}, S_{\lambda}=\left(q_{\lambda \lambda}^{d}\left(\varepsilon_{\lambda}\right)\right)^{-1}, \\
q^{d}(r, \varepsilon)=\left(1-\frac{d \Delta^{d}(\varepsilon)}{d \varepsilon}\right) f(r) .
\end{array}
$$

From relationships (19) follows, that another source of unitarity violation is an energy dependence of the real (dispersive) part of the energy-averaged single-quasiparticle self-energy term. This violation can be eliminated by the use of the modified optical-model GF (compare with Eq. (15)):

$$
g_{(\lambda)}^{m}\left(r, r^{\prime}, \varepsilon\right)=\left(q^{d}(r, \varepsilon)\right)^{1 / 2} g_{(\lambda)}\left(r, r^{\prime}, \varepsilon\right)\left(q^{d}\left(r^{\prime}, \varepsilon\right)\right)^{1 / 2} .
$$

The modified one-hole strength $S_{\lambda}^{m}$ is close to unity, but the width $\Gamma_{\lambda}^{m}=\Gamma_{\lambda}$ is not changed.

Using available experimental data concerned with the energy and total width of neutron and proton one-hole states in the above-mentioned parent nuclei and also the dispersive relationship of Eq. (16), 
we deduced within the developed unitary version of the SQDOM the quantities $W(\varepsilon), \Delta^{p}(\varepsilon), \Delta^{d}(\varepsilon)$ [4]. We found, in particular, that differently from the model of Ref. [17], the contribution of the dispersive part in the optical-model potential is markedly suppressed. An example is given in Fig. 4 in applying to the proton subsystem of the ${ }^{208} \mathrm{~Pb}$ parent nucleus. Also in Ref. [4], properties of deep one-hole states in the ${ }^{132} \mathrm{Sn}$ parent nucleus are predicted.

In conclusion, the unitary version of the single-quasiparticle dispersive optical model is proposed in applying to the description of deep one-hole states in medium-heavy mass spherical nuclei. The next stage of this investigation consists in extension of the model on continuum problems. The respective problem is under consideration.

\section{Acknowledgements}

The Author is very grateful to M. L. Gorelik, S. Yu. Igashov, G. V. Kolomiytsev, S. Shlomo, and B. A. Tulupov for fruitful collaboration. Special thanks to V. G. Zelevinsky for many valuable remarks and G. V. Kolomiytsev for kind help in preparing the manuscript.

This work is supported by the Russian Foundation for Basic Research under grant No. 15-0208007-a and the Competitiveness Program of NRNU "MEPhI".

\section{References}

[1] A.B. Migdal, Theory of finite Fermi systems and applications to atomic nuclei (Interscience, 1967)

[2] V.V. Sokolov, V. Zelevinsky, Physical Review C 56, 311 (1997)

[3] M.H. Urin, Physical Review C 87, 044330 (2013)

[4] G.V. Kolomiytsev, S.Y. Igashov, M.H. Urin, Physics of Atomic Nuclei 80, 614 (2017)

[5] S. Shlomo, G. Bertsch, Nuclear Physics A 243, 507 (1975)

[6] M.H. Urin, Nuclear Physics A 811, 107 (2008)

[7] B.A. Tulupov, M.H. Urin, Physical Review C 90, 034613 (2014)

[8] M.L. Gorelik, S. Shlomo, B.A. Tulupov, M.H. Urin, Nuclear Physics A 955, 116 (2016)

[9] G.V. Kolomiytsev, S.Y. Igashov, M.H. Urin, Physics of Atomic Nuclei 77, 1105 (2014)

[10] B. Tulupov, M.H. Urin, Physics of Atomic Nuclei 72, 737 (2009)

[11] G.F. Bertsch, P.F. Bortignon, R.A. Broglia, Reviews of Modern Physics 55, 287 (1983)

[12] M.L. Gorelik, S. Shlomo, B.A. Tulupov, M.H. Urin, arXiv preprint arXiv:1710.04609 [nucl-th] (2017)

[13] S.E. Muraviev, M.H. Urin, Nuclear Physics A 572, 267 (1994)

[14] D.H. Youngblood, Y.W. Lui, J. Button, M.R. Anders, M.L. Gorelik, M.H. Urin, S. Shlomo, Physical Review C 88, 021301 (2013)

[15] M.L. Gorelik, V.S. Rykovanov, M.G. Urin, Physics of Atomic Nuclei 73, 1997 (2010)

[16] G.V. Kolomiytsev, M.L. Gorelik, M.H. Urin, arXiv preprint arXiv:1712.05146 [nucl-th] (2017)

[17] C. Mahaux, R. Sartor, in Advances in nuclear physics (Springer, 1991), pp. 1-223

[18] S. Shlomo, V.M. Kolomietz, H. Dejbakhsh, Physical Review C 55, 1972 (1997) 